How to reference this article Acerra, E. (2017). Letteratura per I'infanzia e applicazioni: adattamenti ipermediali di opere classiche e albi contemporanei. Italica Wratislaviensia, 8(1), 11-28.

DOI: http://dx.doi.org/10.15804/IW.2017.08.01

\author{
Eleonora Acerra
}

University of Montpellier

eleonora.acerra@umontpellier.fr

\title{
LETTERATURA PER L'INFANZIA E APPLICAZIONI: ADATTAMENTI IPERMEDIALI DI OPERE CLASSICHE E ALBI CONTEMPORANEI
}

\section{CHILDREN'S LITERATURE IN THE WORLD OF APPS: HYPERMEDIA ADAPTATIONS OF CLASSIC AND CONTEMPORARY PICTUREBOOKS}

\begin{abstract}
This analysis aims to describe the main features of hypermedia literary apps for children by considering a corpus of adaptations of classic and contemporary picturebooks. In particular, it highlights the multimodal nature that defines hypermedia texts and their meanings, as well as the interactivity that programs different degrees of readers' involvement and the various ways of using and exploring the virtual space of touchscreens. By describing some hypermedia features deployed in a selection of classic texts, I focus on the hypermedia adaptations of three contemporary Italian picturebooks: Iole, la balena mangiaparole by Gioia Marchegiani, Amico ragnolo by Gloria Francella, and Love, the App by Alberto Vanni. These were chosen to illustrate the significant literary use of several textual materials, the implementation of different types of interactivity, and the recreation of the material characteristics of the print edition through the virtual page. Founded on the appropriation and re-interpretation of the source, the adaptations considered here show a mix of literary intents and digital technologies, while highlighting the substantial homogeneity of the hypermedia strategies used in adapting classic and contemporary texts.
\end{abstract}

Keywords: hypermedia, apps, children's literature, adaptation, picturebooks 


\section{INTRODUZIONE}

Si potrebbe descrivere la storia della letteratura per l'infanzia come un lungo percorso in cui testi, immagini, formati e supporti di lettura si sono intrecciati con l'evolversi del modo di concepire l'infanzia, l'educazione, la valenza sociale e pedagogica attribuita al libro, al tessuto iconografico e al testo letterario, producendo, di volta in volta, una svolta nella scelta di temi e codici. In questo percorso fluido di storie e testi, gli store di applicazioni si configurano come il più recente ecosistema di sviluppo di universi narrativi, all'interno del quale vanno definendosi nuovi modi di ricezione dell'opera letteraria, basati sulla natura ipermediale della composizione e sulla dinamica multimodale (Jewitt e Kress, 2003; Lebrun, Lacelle e Boutin, 2012) determinata dalla pluralità di materie testuali in uso (Saemmer, 2007).

Concepite e organizzate secondo logiche squisitamente commerciali, le piattaforme di applicazioni restano, tuttavia, luoghi da cui osservare i fatti letterari con precauzione. Sui loro scaffali, l'imponente offerta dedicata all'infanzia (cf. Zytnik, 2014) presenta contenuti ludo-educativi, applicazioni di intrattenimento, prodotti derivati dell'industria multimediale e creazioni letterarie, liberamente categorizzati da editori e sviluppatori secondo valutazioni spesso legate agli orientamenti del mercato $\mathrm{o}$ al segmento di pubblico che si desidera raggiungere ${ }^{1}$.

In questo ambiente composito, la letteratura occupa uno spazio modesto ma significativo e, sostenuta dal crescente riconoscimento accordatole dalle istituzioni e dagli spazi di cultura ufficiali² ${ }^{2}$, si ritaglia un

1 Per questa ragione, contenuti ludici appaiono di frequente nella categoria consacrata alla lettura, mentre altri prodotti, benché simili per composizione, funzionalità e pubblico di destinazione, sono talvolta considerati giochi, talaltra libri. $\mathrm{Si}$ vedano per esempio le creazioni di DeAgostini Libri, Fiabe Preziose: Cappuccetto Rosso e Fiabe Preziose: La Sirenetta: sebbene siano entrambe adattamenti di classici $\mathrm{e}$, formalmente, una riproduzione seriale l'una dell'altra, la prima è classificata come prodotto d'Intrattenimento, l'altra come Libro.

2 Si pensi ai premi dedicati alle opere digitali nelle fiere del libro per l'infanzia, come il Bologna Ragazzi Digital Award o il riconoscimento delle Pépites Numériques al Salone del libro di Montreuil. 
ruolo di effervescente sperimentazione poetica. Creazioni "native del digitale", produzioni transmediali e crossmediali (Antoniazzi, 2015) si affiancano a un più ricco catalogo di trasposizioni e adattamenti di testi classici e di albi contemporanei, di cui ci si occuperà in questo articolo. Spesso molto diverse per qualità e legittimità culturale, queste opere mostrano, insieme all'inestinguibile fecondità delle pratiche adattative, analogie importanti con l'offerta editoriale tradizionale ${ }^{3}$ : nelle librerie digitali, come in quelle cartacee, accanto a una schiera di versioni edulcorate delle opere d'origine e a riscritture in cui il legame col testo di partenza è appena visibile, si segnalano adattamenti più raffinati, frutto di una profonda riflessione sull'opera di partenza, di cui si riappropriano, selezionandone alcuni passaggi chiave e restituendoli in chiave intersemiotica (Dusi e Neergard, 2000; Eco, 2012), alla luce di un preciso progetto letterario. Ne Le Horla dell'Apprimerie (2014), trasposizione integrale dell'omonimo racconto di Maupassant, ad esempio, animazioni, immagini e giochi interattivi contribuiscono a delineare, con le loro atmosfere inquietanti, una delle tante, possibili interpretazioni dell'ambigua follia del protagonista. Altre creazioni si concentrano piuttosto sulla rielaborazione di elementi costitutivi del formato cartaceo della matrice: ne è un esempio Moi, j'attends di France Television (2013), adattamento dell'albo eponimico di Serge Cali e David Bloch, in cui viene restituito, oltre all'universo poetico dell'originale, il formato all'italiana dell'edizione cartacea: trasformata in un flusso di schermi, la pagina ora "scorre" da sola, sollecitata dall'azione del lettore su un filo polimorfo, metafora della vita, che sarà correttamente inteso e manipolato soltanto investendo le proprie conoscenze e la propria sensibilità ${ }^{4}$. In altri casi, gli adattamenti ipermediali sembrano realizzare alcune potenzialità presenti soltanto allo stato embrionale nelle edizioni cartacee È così ad esempio per Un jeu (2011), sviluppato a partire dall'opera di Hervé Tullet, e per Les jeux du livre des bruits (2011), ispirato dal te-

3 Per uno studio dell'attuale offerta editoriale per l'infanzia, cf. Antoniazzi in Beseghi e Grilli, 2014.

4 Per una descrizione più ampia dell'opera si veda Acerra, 2016. 
sto di Soledad Bravi ${ }^{5}$ : dando rispettivamente movimento alle figure del primo e voce alle creature del secondo, le due applicazioni sembrano assecondare l'invito all'interazione e alla riproduzione sonora intrinseco alle due opere. Quando non si limitano ad assecondare le attese del marketing, le applicazioni mostrano insomma strutture e progetti precisi, ricerche estetiche e poetiche. Formate di elementi ipermediali e di dispositivi interattivi, costruite secondo un'idea specifica delle modalità di lettura e del loro "lettore implicito", esse richiedono strumenti d'analisi letteraria specifici, capaci di rendere conto della pluralità delle loro risorse semiotiche, dei loro spazi e delle loro dinamiche, ma anche delle peculiarità dovute all'utilizzo di strumenti e supporti tecnologici. Studi recenti mostrano infatti che la ricezione del testo ipermediale, lungi dall'innestarsi in maniera spontanea e secondo costanti universali nei giovani lettori, è al contrario condizionata dai profili e dalle pratiche culturali (digitali e non) di ciascuno, dai contesti socio-familiari di provenienza, dal proprio immaginario sulla lettura (Ramada Prieto e Reyes López, 2015), ma anche dalla maggiore o minore disponibilità a rivestire il ruolo di agenti attivi, immersi in percorsi letterari dinamici, ludici e talvolta labirintici ${ }^{6}$. Sebbene numerose analisi condotte nel campo della psicologia cognitiva abbiano provato il carattere generalizzabile dei processi di comprensione del testo narrativo, a prescindere dalle modalità con cui viene fruito (multimediali, audiovisuali o auditive) (Kendeu et al., 2005; Van den Broek et al. 2005), le prime ricerche dedicate alla letteratura ipermediale mostrano reazioni variabili, tanto rispetto alla ricezione e all'interpretazione di contenuti specifici che, più in generale, all'apprezzamento dell'esperienza estetica vissuta. L'entusiasmo spontaneo dei giovani per gli schermi e per i prodotti digitali, nell'ambito domestico come in quello scolastico (Aliagas e Margallo, 2015), tende a scemare e a deviare verso usi meramente ludici in assenza di un adulto che ne guidi le osservazioni e incoraggi gli scambi

5 Per un'analisi delle caratteristiche degli albi senza parole, cf. Mirandola in Hamelin, 2012.

6 In ambito scolastico, occorrono variazioni imputabili a tratti caratteriali, al comportamento in gruppo e al grado di sviluppo delle abilità motrici (Real e Correro, 2015, p. 181). 
verbali (Real e Correro, 2015, p. 185). La comprensione, spesso valutata comparando i risultati di due gruppi di lettori, gli uni confrontati a storie lette su tablet, gli altri su supporto librario, pur non presentando rilevanti scarti statistici (Sheppard, 2014), sembra strettamente connessa, oltre che alle competenze di ciascuno ${ }^{7}$, alle caratteristiche delle opere proposte. Le interazioni e le attività ricreative $i$ cui scopi narrativi paiono più opachi e con meccaniche di visualizzazione troppo prevedibili o aleatorie, sono ad esempio percepite come fattori di distrazione e sembrano ridurre, insieme alla comprensione, l'investimento e il coinvolgimento emozionale dei lettori, che pure le indicano sistematicamente tra le ragioni della piacevolezza e dell'intensità dell'incontro con il testo ipermediale (Manresa, 2015: 111). Per questo motivo, le prime griglie di valutazione delle opere narrative per l'infanzia si sforzano di stabilire criteri per la costituzione di corpus validi dal punto di vista letterario ed ipermediale (Yokota, 2014) e offrono al contempo una prospettiva sulle principali tendenze della produzione applicativa, al momento caratterizzata soprattutto dall'interpolazione extra-letteraria, l'onnipresenza dell'interattività e la preferenza per strutture narrative semplici (Turrión Penelas, 2015, p. 91).

Pur non entrando nel merito della loro pertinenza pedagogico-cognitiva, in questo articolo ci si soffermerà su alcune caratteristiche delle applicazioni di letteratura per l'infanzia, considerate attraverso lo studio di un corpus di adattamenti di opere classiche e contemporanee. Senza ambire all'esaustività e nella consapevolezza che le repentine variazioni di formati e supporti renderanno presto obsolete le nostre conclusioni, ci limiteremo ad analizzare gli elementi che sembrano, ad oggi, più significativi: la pluralità di materie semiotiche che compongono il tessuto narrativo; i differenti gradi di interattività, che coinvolgono il lettore e ne sollecitano, a livelli diversi, l'empatia; le diverse possibilità nel trattamento della pagina e dei suoi spazi. Muovendo dall'esame dei dispositivi in atto in una rosa di trasposizioni di testi classici in cui figurano adattamenti e rifacimenti parodici, si passerà all'analisi della

7 I dati mostrano infatti risultati meno soddisfacenti nella comprensione del testo fruito su supporto digitale per i lettori più deboli (Sheppard, 2014). 
produzione contemporanea italiana, descrivendo le versioni ipermediali degli albi di Gioia Marchegiani (Iole, la balena mangiaparole), Gloria Francella (Amico ragnolo) e Alberto Vanni (Love), rispettivamente scelti per illustrare la molteplicità di materie testuali di un'opera ipermediale, i vari livelli di interattività che è possibile programmare e la resa su schermo delle caratteristiche materiali dell'edizione stampata. L'analisi parallela di adattamenti di testi classici e contemporanei permetterà di verificare al contempo la sostanziale omogeneità di strategie e dispositivi ipermediali nelle due categorie di testi, indipendentemente dalla loro diversa legittimità culturale.

La scelta di occuparsi di adattamenti ipermediali ha, infine, un duplice intento. Da un lato, ci sembra offrire un quadro veritiero dell'attuale proposta degli store di applicazioni, ben più prolifici in materia di trasposizioni che di creazioni originali. Dall'altro, permette di iscrivere le opere ipermediali nel solco della tradizione letteraria, creando un filo rosso tra codici, storie e personaggi, che si spera possa ricondurre ogni sterile contrapposizione tra libro di carta e libro virtuale a un'osservazione puntale di forme, stili, testi e immagini, cui queste opere hanno diritto.

\section{ADATTAMENTI DI CLASSICI E IPERMEDIALITÀ}

La trasposizione di classici dell'infanzia in opere ipermediali appartiene a quella tipologia di processi adattativi che ha permesso ad opere e testi, nel corso dei secoli, di migrare da una forma semiotica all'altra, producendo una moltitudine di oggetti secondi, attraverso i quali sfuggire ai capricci della trasmissione testuale. Versioni ridotte, allungate, aggiornate, talvolta riscritte o riformulate per nuovi uditori ${ }^{8}$, ma anche trans-finzioni (Saint-Gelais, 2011) e ipertesti (Genette, 1982), popolano il catalogo degli store e fanno dell'adattamento ipermediale, come di ogni adattamento, un lavorio "di lettura e d'interpretazione" (Louichon, 2015a), in cui storie e personaggi prendono sfumature inedite, svincolandosi, nei casi più estremi, dalle pagine in cui hanno trovato la luce,

8 Cf. Helbo, 1997. 
per intraprendere avventure del tutto indipendenti dalle vicende originarie ${ }^{9}$.

In questo panorama di produzioni, persiste un'importante disparità tra uno sparuto gruppo di trasposizioni integrali, come Le Horla de L'Apprimerie, e un ben più ricco catalogo di versioni abbreviate o riassunte, la cui esiguità è in parte imputabile agli elevati costi di produzione, difficilmente sostenibili per le case editrici di piccole dimensioni e di rado compensati da vendite adeguate. Il Pinocchio di Elastico (2011), ad esempio, nonostante la fine selezione dei passaggi trasposti, riduce $i$ trentasei capitoli dell'originale a una ventina di pagine-schermo, lasciando ad animazioni, immagini e contenuti sonori il compito di sopperire alla laconicità del testo con un insieme di richiami evocativi all'opera di Collodi. Nella seconda pagina-schermo dell'applicazione, ad esempio, gli effetti animati e interattivi illustrano l'insolenza del burattino appena costruito: non appena il lettore lo sfiora, Pinocchio scopre il suo lungo naso, comincia a muovere gli occhi e a ridere con impertinenza, proprio come faceva nel testo di Collodi, cui si allude senza riportarlo. Voyage au centre de la terre, un'altra creazione de L'Apprimerie (2014), opera tagli ugualmente drastici, pur preferendo accompagnare con dispostivi ipermediali il testo trasposto, piuttosto che quello assente: nel passo in cui i protagonisti scendono verso l'oscurità del centro della terra (p. 37), la pagina, minacciosamente scura, s'illumina appena per permettere la lettura, mentre il testo, altrettanto scuro, come seguendo la marcia serpentina dei due viaggiatori, continua a sprofondare zigzagando verso il fondo dello schermo.

Altrettanto varie risultano le modalità di trattamento dell'opera classica. Si vedano, a titolo esemplificativo, alcune delle innumerevoli declinazioni di Cappuccetto Rosso presenti negli store, in cui si riconoscerà, tra l'altro, il gusto contemporaneo per il capovolgimento degli stereotipi della lettura e per la riscrittura parodica, nonché l'attenzione a problematiche del mondo odierno, come l'impatto ecologico e la sofferenza animale. Nella trasposizione a-testuale sviluppata da Brian

9 Cf. Audet e Saint-Gelais, 2000; Louichon, 2015b; Saint-Gelais, 2011; Salviati, 2002. 
Main (2012), la narrazione è affidata a una sequenza iconografica e a una serie di fumetti illustrati che, insieme a un repertorio di suoni e rumori, danno voce ai personaggi e ai loro sentimenti. In un'atmosfera dai sapori manga, il racconto tradizionale, pur seguito a grandi linee, trova importanti variazioni soprattutto nel finale ${ }^{10}$, assicurando al lettore una chiusa comica, in cui la piccola protagonista verrà espulsa dal ventre del lupo alla fine di una rocambolesca rissa con un cacciatore tutto muscoli. Un'analoga disinvoltura adattativa si riscontra nella riscrittura in chiave moderna e parodica proposta dello studio Blue Quoll (2011), in cui un'inedita Cappuccetto Rosso si batte (a colpi di scacchi) per preservare dalla voracità di un lupo vegetariano il suo prezioso cesto di biscotti allo zenzero. In quest'opera, priva di ogni eco al testo moralizzatore della tradizione, le interazioni, dispiegandosi su un tessuto iconografico sostanzialmente statico, permettono l'apparizione di un centinaio di nuvolette di testi nascoste sullo schermo: sotto l'imput del lettore, esse rivelano definizioni ironiche, descrizioni curiose e scorci di dialoghi che insistono sull' aspetto leggero dell'opera ${ }^{11}$. La distanza dalle versioni di Perrault o dei fratelli Grimm è, infine, ancor più marcata nella riscrittura prodotta dallo studio Bobaka, il cui universo ipermediale e ludico presenta una Cappuccetto Verde, ecologista e appassionata di yoga, intenta a convincere un lupo sempre più affamato dei benefici di uno stile di vita sano e di un'alimentazione che non metta a rischio la vita degli altri animali.

***

In quanto creazioni digitali, le opere del nostro corpus si iscrivono sulle orme della letteratura digitale precedente, con cui condividono in primis il carattere informatico della composizione, visibile tanto nell'or-

10 In questa versione, ad esempio, la nonna di Cappuccetto Rosso non viene divorata dal lupo ma, dopo essersi nascosta in un armadio, si reca personalmente a cercare aiuto dal cacciatore.

11 Si noti che tanto il titolo quanto l'icona dell'applicazione sottolineano le differenze rispetto all'opera classica, mettendo in primo piano la figura del lupo anziché quella di Cappuccetto Rosso. 
ganizzazione degli elementi nello spazio che nella programmazione dei differenti percorsi interattivi. Ciò equivale a dire che nelle applicazioni di letteratura per l'infanzia, come nel resto nella produzione digitale, anche quando la navigazione e l'interazione sembrano assecondare i movimenti più liberi e improvvisati dell'interlettore, ogni azione o gesto è stato in realtà già previsto a monte, riducendo l'infinità di schemi virtualmente percorribili a un ventaglio di percorsi predefiniti, selezionati unendo al progetto letterario e adattativo dell'opera osservazioni e studi ergonomici e psico-cognitivi, volti ad ottimizzare l'esperienza di lettura e la manipolazione dello schermo.

Come altre composizioni ipermediali, anche le applicazioni per l'infanzia oggetto di questo articolo si compongono di media diversi, e in particolare di testi, suoni, video e immagini animate (Cotton e Oliver, 1993; Delany e Landow, 1991) che, pur conservando le proprie peculiarità semiotiche, co-creano un filo narrativo unico, da leggere e interpretare con un'analisi simultanea e parallela dei vari elementi. In continuità con altri testi ipermediali, inoltre, queste creazioni prediligono alla trasparenza mediatica tipica delle opere digitali di prima di generazione l'uso di numerose entità testuali, i cui "segni di mediazione", tesi a riprodurre la ricchezza dell'apparato sensoriale umano (Bolter e Grusin, 2000 , p. 34), contribuiscono trasformare l'atto della lettura in un momento poli-sensoriale irrepetibile.

Altre caratteristiche mostrano invece rotture con la precedente tradizione letteraria digitale. È in particolar modo abbandonata quella ricerca di spaesamento, spesso ottenuta tramite uno sviluppo narrativo per nodi ipertestuali, che sbalzava il lettore da un blocco di lexia ${ }^{12}$ (Barthes, 1970) all'altro senza offrirgli una visione d'insieme sul percorso effettuato. Ad oggi, l'effetto di straniamento è costantemente temperato dal ricorso ad interfacce che permettono di situare il proprio livello di avanzamento nella lettura; la navigazione nell'opera si organizza intorno a percorsi lineari e non tabulari, evitando di interferire con la fine e la

12 Si ricorda che a partire dalla nozione di lexia di Barthes, Landow poté definire l'ipertesto come una struttura composta di blocchi di testo (lexia) e collegamenti elettronici (1992). 
sequenzialità degli eventi, riconosciute da Gervais come le "strutture portanti" del testo (1999, p. 116).

La sperimentazione formale resta tuttavia centrale e si concentra sulle diverse possibilità di organizzazione testuale e sulle dinamiche di manipolazione offerte dai dispositivi tattili. Concepite per servirsi della tecnologia e della mobilità dei tablet, le opere ipermediali plasmano, infatti, gli spazi dello schermo, divenuto duttile e virtualmente privo delle costrizioni dei formati cartacei. Piegata ad esigenze narrative ogni volta diverse, la pagina digitale si sfoglia ora verso l'altro ora verso il basso, si scorre con un dito, si attiva tramite un'interazione o, ancora, ricrea i gesti tipici della lettura di un audiolibro. Al contempo, ipermedialità e interattività, prevedendo tipologie diverse d'interventi sullo schermo, giocano con l'alternanza di focalizzazioni del lettore e contribuiscono a determinare i vari gradi della sua implicazione. In opere come Pinocchio (Elastico, 2011), ad esempio, il lettore si trova ora ad affiancare il burattino nelle sue disavventure, ora ad osservarlo da una posizione distante e protetta, da cui potrà intervenire sulle scene rappresentate soltanto come agente esterno, privo di ogni reale capacità di modificare l'esito delle vicende, il cui sviluppo resta confinato in uno spazio narrativo inaccessibile.

\section{LA LETTERATURA PER L'INFANZIA SUGLI STORE ITALIANI}

Gli store di applicazioni italiani non mostrano variazioni significative rispetto alla produzione internazionale né per quanto riguarda la distribuzione di adattamenti tra testi classici e contemporanei, né per le strutture compositive e l'organizzazione del tessuto testuale, le cui possibilità appaiono, anche in questo corpus, innumerevoli. Restano prevalenti gli adattamenti e le trasposizione di classici della letteratura, mentre la produzione contemporanea, meno rappresentata, si serve dell'ipermedialità e dell'interattività soprattutto per restituire il linguaggio poetico e minimalista tipico dell'albo odierno. Sulla scia della produzione editoriale cartacea, gli adattamenti ipermediali di opere contemporanee prediligono, infatti, temi complessi e aperti a rappresentazioni diverse, lasciando volentieri al lettore il compito di colmare gli spazi bianchi del testo 
e d'interpretare le ambiguità o il non detto di pagine spesso reticenti. Le tre opere che seguono si offrono quali esempi di quella scrittura poetica ed essenziale che "scava nell'intimo" (Blezza Picherle 2004, p. 247), mostrando la realtà screziata di vissuti difficili e talvolta dolorosi, attraverso un linguaggio che non disdegna la vaghezza, l'espressione metaforica e figurata (cf. Blezza Picherle 2004, pp. 264-271). Al contempo, tramite le caratteristiche del testo digitale, queste opere moltiplicano il fascino della narrazione, plasmando suggestioni che danno a vedere ora gli stati d'animo e le emozioni dei personaggi, ora alcuni passaggi del testo, ora l'intenso spessore della pagina digitale. Tramite l'adattamento dell'albo di Gioia Marchegiani, Iole, la balena mangiaparole (2013), s'illustrerà un utilizzo di materie testuali tale da esaltare la poeticità dell'originale; l'opera di Gloria Francella, Amico ragnolo (2014), mostrerà come l'interattività possa stimolare il coinvolgimento del lettore e l'esplorazione dello schermo; infine, la trasposizione di Love, celebre albo di Gian Berto Vanni, presenterà una ricostruzione della struttura e della consistenza della pagina cartacea.

\section{LE FORME DELLA MATERIA: UN'ESPERIENZA MULTIMODALE CON IOLE, LA BALENA MANGIAPAROLE}

Attraverso Iole la balena mangiaparole, opera dell'artista romana Gioia Marchegiani (2012), si penetra direttamente in quella mistione mediatica polisemica tipica della produzione ipermediale: suoni, giochi tipografici, immagini e componenti testuali, stabilendo rapporti diversi (illustrativi, metaforici o evocativi), concorrono alla costituzione del tessuto testuale, sollecitando al contempo la partecipazione del lettore. La storia, delicata e surreale, di una balena che si nutre e crea storie con le parole scartate da un poeta, è infatti adattata affidando ai tratti virtuali dell'acquarello, a una serie di accorgimenti tipografici e a leggere interazioni, la resa dell'atmosfera poetica e suggestiva della composizione originale. Mentre il testo, relegato nella sezione inferiore della pagina, compare soltanto al tocco del lettore, l'illustrazione, preponderante, descrive con finezza i protagonisti, rendendone cromaticamente leggibili emozioni e umori: rossa, nelle ore di gioia vissute nell'attesa del poeta e delle sue 
parole, la balena diviene via via più scura al momento della sua scomparsa, che la lascia digiuna e sconsolata, per poi recuperare il suo colore deciso dopo l'apporto consolatorio degli amici. L'interazione, conducendo il lettore dallo schermo fin negli abissi, permette di affiancare i rotondi movimenti della balena, per esempio facendo sì che spalanchi la bocca per accogliere il cibo gettato via dal poeta. Un flusso di parole discende allora, con una lentezza che l'animazione e l'accompagnamento musicale non mancano di rilevare, dal poeta verso il mare, per approdare infine, liquido e leggero, sul fondale. La disposizione e l'orientamento delle parole, prediligendo forme non convenzionali e restando sensibili ai gesti e alle aggiunte del lettore, canalizzano l'attenzione sul valore cruciale della parola nella composizione. Ogni termine scartato dal poeta o emesso dalla balena ha una grandezza, uno spessore e un preciso tempo di scorrimento; può animarsi, spostarsi o ruotare, con effetti che evocano fedelmente la realtà degli spostamenti in un ambiente acquatico. Le parole, nel mare, galleggiano o affondano a un ritmo dolce e pesante, in cui risultano tangibili e carichi di significato l'attrito dell'acqua e il "peso della poesia".

\section{VARI GRADI D'INTERATTIVITÀ: UN'ESPLORAZIONE DEI POSSIBILI CON AMICO RAGNOLO}

La nozione di interattività, complessa e molto studiata ${ }^{13}$, invita a riconsiderare i vari livelli con cui l'opera ipermediale può programmare l'azione del lettore-utilizzatore sullo schermo. Seguendo la distinzione di Ryan (2015), che postula una doppia dicotomia tra interattività interna (quando il lettore-utilizzatore agisce come un membro dell'universo della finzione) ed esterna (quando, al contrario, controlla lo spazio finzionale dall'esterno, come un deus ex machina), nonché tra interattività esploratoria (quando l'azione del lettore-utilizzatore non ha intenzioni

13 Si pensi ad Andersen (1990), per il quale l'interattività implica la possibilità di alterare fisicamente il discorso al fine di produrre nuovi significati; ad Aarseth (1997), per cui implica una co-azione di apporti umani e meccanici; o ancora a Murray (1997) che, preferendovi il termine di agentività, la definisce come un insieme di azioni significanti capaci di alterare il mondo della finzione. 
creative né un'influenza duratura sullo sviluppo narrativo) ed ontologica (quando invece produce effetti permanenti sul testo e sulla narrazione), si considererà la storia di Amico ragnolo come un esempio di interventi esploratori esterni, finalizzati a coinvolgere il lettore tanto nelle peripezie dei protagonisti quanto nello spazio dello schermo. L'improbabile amicizia tra un ragno e un papero immaginata da Gloria Francella (2012) si svela infatti leggendo i testi e le illustrazioni, ma anche assecondando le interazioni previste dall'opera. Principalmente di due tipi, le azioni richieste permettono da un lato l'avanzamento narrativo, dall'altro l'intervento fisico del lettore sulle vicende narrate. Sfiorando il ragno, l'interlettore ne vedrà le scaltre abilità di tessitore, toccando il cielo ne osserverà i colori cambiare secondo il periodo del giorno e, fendendo lo schermo, proverà il brivido di una sfida a colpi di spade. Altrove, potrà colmare la laconicità del testo "leggendo" gli effetti prodotti dai suoi gesti: è così, ad esempio, nella pagina-schermo in cui il papero, raccontando del coraggio che gli infonde la compagnia dell'amico, scampa all'attacco di un cane grazie al prezioso soccorso di un insetto, la cui presenza sfugge a chi tralasci l'interazione. Al tempo stesso, esortato da indicazioni e richiami espliciti, il lettore è invitato a confrontarsi con la mobilità del supporto: potrà, ad esempio, scuotere lo schermo fino ad avvolgere il papero, troppo agitato, con la tela dell'amico.

\section{LO SPAZIO DELLA PAGINA. TOCCARE L'INVISIBILE CON LOVE, THE APP}

Come anticipato, l'uso di dispositivi ipermediali può spingersi sino alla restituzione dell'aspetto materiale della pagina cartacea. Love, the App, adattamento dell'albo di Gian Berto Vanni e Lowel Siff (1964), ricrea quel montaggio di pagine che, nell'opera originaria, legava magistralmente fori e colori nella narrazione delle vicende di una bambina lasciata nel clima freddo e indifferente di un orfanotrofio. Assemblate secondo un progetto che attribuiva un colore ad ogni stato d'animo, le pagine dell'edizione cartacea, come quelle della trasposizione per iPad, recano su ogni facciata delle aperture polimorfe, intese a creare, col loro scorcio di attese e anticipazioni sulla pagina e i colori successivi, un flusso inin- 
terrotto d'immagini e significati. Incidendosi sulla carta, i fori dell'opera di Vanni illustrano storie e stati d'animo, organizzano lo spazio della pagina e regolano il ritmo della lettura. Così, l'edizione ipermediale, riproducendo con rigore il testo, i colori e le caratteristiche dell'opera fonte, raccoglie la sfida di riprodurre lo spessore materiale e semiotico delle fessure d'origine, usando l'immaterialità dello schermo per moltiplicare metafore e suggestioni. La pagina virtuale, animata e duttile, si strappa, si trascina in lembi sui margini dello schermo o, ancora, si piega per svelare porzioni di testo o illustrazioni nascoste. Nel frattempo, le animazioni danno vita alle metafore dell'opera originaria, lasciando crollare i muri di solitudine che circondano la protagonista o, ancora, facendosi strumento per volare, almeno con la fantasia, oltre gli spazi angusti dell'orfanotrofio in cui vive.

\section{CONCLUSIONI}

L'adattamento ipermediale, qui analizzato attraverso un corpus di trasposizioni di testi classici e contemporanei, mostra alcuni processi tipici delle pratiche adattative. Partendo da un'interpretazione e da una riappropriazione dell'opera-fonte, restituisce infatti una visione dell'opera originaria che è il prodotto simbiotico dello sguardo dell'adattatore e delle potenzialità semiotiche del medium di destinazione.

In queste opere, le caratteristiche proprie del testo ipermediale e l'interattività partecipano alla costruzione di universi narrativi polisemici, in cui ogni singolo elemento veicola messaggi e suggestioni. Azioni e interazioni, modellate per accogliere il progetto adattativo e per rivelarne i percorsi, sembrano costantemente programmate per catturare l'attenzione del lettore, per sollecitarne la cooperazione interpretativa e le emozioni. Affiancando i personaggi, comprendendo le relazioni che legano le diverse risorse semiotiche e determinando l'avanzamento narrativo, egli diviene un nuovo lettore agente, i cui gesti sono previsti per seguire in maniera parallela un disegno letterario e tecnologico.

Sostanzialmente simili nell'adattamento di testi classici e di opere contemporanee, dispositivi ipermediali e interattivi si dispiegano sullo schermo rendendone visibili la duttilità e la plasticità, ampliando l'atto 
di lettura fino a renderlo un'esperienza in cui s'intrecciano testualità multimodali, incontri sensoriali e processi immaginativi.

\section{BIBLIOGRAFIA}

Aarseth, E. J. (1997). Cybertext: Perspectives on Ergodic Literature. Baltimora (USA): Johns Hopkins University Press.

Acerra, E. (2016). Poétique des œuvres hypermédiatiques dans un corpus d'adaptations de littérature pour la jeunesse. Poétiques et esthétiques numériques tactiles: Littérature et Arts. Cahiers virtuels du Laboratoire NT2 n. 8. Disponibile da: http://nt2.uqam.ca/fr/cahiers-virtuels/article/ poetique-des-oeuvres-hypermediatiques-dans-un-corpus-dadaptationsde

Aliagas, C., Margallo, A. M. (2015). iPads, Emergent Readers and Families. In M. Manresa e N. Real (a cura di), Digital Literature for Children. Texts, Readers and Educational Practices (pp. 155-171). Bruxelles: Peter Lang.

Andersen, P. B. (1997). A Theory of Computer Semiotics: Semiotic Approaches to Construction and Assessment of Computer Systems. Cambridge: Cambridge University Press.

Antoniazzi, A. (2015). Dai Puffi a Peppa Pig: media e modelli educativi. Bologna-Roma: Carocci.

Audet, R., Saint-Gelais, R. (a cura di). (2007). La Fiction, Suites et Variations. Québec e Rennes: Éditions Nota bene e Presses universitaires de Rennes.

Barthes, R. (1970). S/Z. Parigi: Éd. du Seuil.

Beseghi, E., Grilli, G. (a cura di). (2014). La letteratura invisibile: infanzia e libri per bambini. Roma: Carocci.

Blezza Picherle, S. (2004). Libri, bambini, ragazzi: incontri tra educazione e letteratura. Milano: Vita e pensiero.

Bolter, J. D., Grusin, R. A. (2000). Remediation: Understanding New Media. Cambridge (USA): The MIT Press.

Bouchardon, S. (2009). Littérature numérique: le récit interactif. Parigi: Hermès Science: Lavoisier.

Cotton, B., Oliver, R. (1993). Understanding Hypermedia: from Multimedia to Virtual Reality. Londra: Phaidon Press.

Delany, P., Landow, G. P. (a cura di). (1991). Hypermedia and Literary Studies. Cambridge (USA): The MIT Press. 
Dusi, N., Neergard, S. (a cura di). (2000). Sulla traduzione intersemiotica. Versus. Quaderni di studi semiotici, nn. 85-87. Bologna: Bompiani.

Eco, U. (2012). Dire quasi la stessa cosa: esperienze di traduzione. Milano: Bompiani.

Faeti, A. (2011). Guardare le figure. Gli illustratori italiani dei libri per l'infanzia. Roma: Donzelli.

Genette, G. (1982). Palimpsestes: la littérature au second degré. Parigi: Éd. du Seuil.

Gervais, B., Xanthos, N. (1999). "L'hypertexte: une lecture sans fin". In A. Vuillemin e M. Lenoble (a cura di), Littérature, informatique, lecture. De la lecture assistée par ordinateur à la lecture interactive (pp. 111-125). Limoges: Pulim.

Jewitt, C., Kress, G. (a cura di). (2003). Multimodal Literacy. New York: Peter Lang.

Hamelin. (2012). Ad occhi aperti. Leggere l'albo illustrato. Roma: Donzelli. Helbo, A. (1997). L'adaptation: du théâtre au cinéma. Parigi: Armand Colin. Kendeu, P., Lynch J. S., Van der Broek, P. W., Espin, C., White, M. e Kremer, K. E. (2005). "Developing Successful Readers: Building Early Comprehension Skills through Television Viewing and Listening". Early Childhood Education Journal, n. 33, 91-98.

Landow, G. P. (1992). Hypertext: the Convergence of Contemporary Critical Theory and Technology. Baltimora (USA): Johns Hopkins University Press.

Lebrun, M., Lacelle, N., Boutin, J. F. (a cura di). (2012). La littératie médiatique multimodale: de nouvelles approches en lecture-écriture à l'école et hors de l'école. Québec: Presses de l'Université du Québec.

Louichon, B. (2015a). "Le patrimoine littéraire : un enjeu de formation". Tre$m a$, n. 43, 22-31.

Louichon, B. (2015b). "Le patrimoine: du passé dans le présent". In M. F. Bishop e A. Beladjin (a cura di), Ecole et patrimoines littéraires (pp. 94-107). Parigi: Champion.

Manresa, M. (2015). "Traditional Readers and Electronic Literature. An Exploration of Perceptions and Readings of Wigital Works". In M. Manresa e N. Real (a cura di), Digital Literature for Children. Texts, Readers and Educational Practices (pp. 105-120). Bruxelles: Peter Lang.

Murray, J. H. (1997). Hamlet on the Holodeck: the Future of Narrative in Cyberspace. Cambridge (USA): The MIT Press. 
Ramada Prieto, L., Reyes López, L. (2015). Digital Migrations: Exploratory Research on Children's E-Lit Reading Profiles. In M. Manresa e N. Real (a cura di), Digital Literature for Children. Texts, Readers and Educational Practices (pp. 121-136). Bruxelles: Peter Lang.

Real, N., Correro, C. (2015). Digital Literature in Early Childhood. Reading Experiences in Family and School Contexts. In M. Manresa e N. Real (a cura di), Digital Literature for Children. Texts, Readers and Educational Practices (pp. 173-189). Bruxelles: Peter Lang.

Ryan, M.-L. (2015). Narrative as Virtual Reality: Immersion and Interactivity in Literature and Electronic Media. Baltimora (USA): Johns Hopkins University Press.

Saemmer, A. (2007). Matières textuelles sur support numérique. SaintEtienne: Publications de l'Université de Saint-Etienne.

Saint-Gelais, R. (2011). Fictions transfuges: la transfictionnalité et ses enjeux. Parigi: Éd. du Seuil.

Salviati, C. I. (2002). Raccontare destini. La fiaba come materia prima dell'immaginario di ieri e di oggi. Torino: Einaudi.

Sheppard, D. (2014). "Reading with iPads - the Difference Makes a Difference". Education Today, n. 3, 12-15.

Terrusi, M. (2012). Albi illustrati. Leggere, guardare, nominare il mondo nei libri per l'infanzia. Roma: Carocci.

Turrión Penelas, C. (2015). "Electronic Literature for Children. Characterising Narrative Apps (2010-2014)”. In M. Manresa e N. Real (a cura di), Digital Literature for Children. Texts, Readers and Educational Practices (pp. 87-102). Bruxelles: Peter Lang.

Yokota, J., William, T. H. (2014). Picture Books and the Digital World: Educators Making Informed Choices. The Reading Teacher, n. 68.8, 577-585.

Van den Broek, P., Kendeou, P., Kremer, K., Lynch, J. S., Butler, J., White, M. J. e Lorch, E. P. (2005). "Assessment of Comprehension Abilities in Young Children». In S. Stahl e S. Paris (a cura di), Children's Reading Comprehension and Assessment. Center for the Improvement of Early Reading Achievement (pp. 107-130). Mahwah, NJ: Lawrence Erlbaum Associates.

Zytnik, M. (2014, settembre 29). School Report for Apple App Store and Google Play [Blog] Disponibile da: https://www.adjust.com/company/ overview/2014/09/29/kids-apps-report-apple-itunes-google-play/ 


\section{Opere ipermediali}

Amico ragnolo, Five5ifty, 2014.

Cappuccetto verde, Boboka, 2015.

I can't wait, France Télévision, 2013.

Le Horla, L'Apprimerie, 2014.

Iole, la balena mangiaparole, Giangiacomo Feltrinelli Editore, 2013.

Les jeux du livre des bruits, Europa Apps, 2011.

Lil'Red, Brian Main, 2012.

Love, the App, Pablo Curti, 2014.

Mr. Lupo e i dolcetti allo zenzero, Blue Quoll, 2012.

Pinocchio, Elastico, 2011.

Voyage au centre de la terre, L'Apprimerie, 2012.

Le Horla, L'Apprimerie, 2014.

Un jeu, Bayard, 2011.

\section{Edizioni cartacee}

Bravi, S. (2004). Le livre des bruits. Parigi: Loulou et Ecole des loisirs.

Cali, D., Bloch, S. (2005). Moi, j'attends... Parigi: Sarbacane.

Collodi, C. (1977). Le avventure di Pinocchio. Milano: Ed. A. Vallardi. A. Garzanti.

Francella, G. (2013). Amico ragnolo. Firenze - Milano: Fatatrac.

Marchegiani, G. (2015). Iole, la balena mangiaparole. Milano: Gribaudo.

Siff, L., Vanni, G.B. (1964). Love, the App. New York: George Braziller.

Tullet, H. (2010). Un livre. Dijon: Talant: Les doigts qui rêvent.

Riassunto: Considerando un corpus di adattamenti di opere classiche e di albi contemporanei italiani, il lavoro si propone di determinare alcune caratteristiche della letteratura ipermediale per l'infanzia. Saranno in particolar modo esaminati i tessuti compositi e multimodali che costituiscono il testo ipermediale e permettono di costruirne il senso; l'interattività, che prevede diversi gradi di coinvolgimento del lettore; alcuni modi di utilizzo ed esplorazione degli spazi virtuali degli schermi tattili. Muovendo dall'esame dei dispositivi in opera in una rosa di trasposizioni di testi classici, ci si soffermerà in particolare sulle versioni ipermediali degli albi contemporanei di Gioia Marchegiani (Iole, la balena mangiaparole), Gloria Francella (Amico ragnolo) e Alberto Vanni (Love, the App), scelti rispettivamente per illustrare la molteplicità di materie testuali, il ricorso a diverse tipologie di elementi interattivi e un uso degli spazi della pagina virtuali tale da restituire le caratteristiche materiali delle edizioni cartacee. Fondati sull'appropriazione-interpretazione di elementi testuali e sull'utilizzo a fini narrativi delle possibilità tecnologiche fornite dagli schermi tattili, gli adattamenti ipermediali qui considerati permettono di verificare al contempo l'ipotesi della sostanziale omogeneità di strategie e dispositivi ipermediali in uso nelle due categorie di testi, indipendentemente dalla loro diversa legittimità patrimoniale.

Parole chiave: ipermedialità, applicazioni, letteratura per l'infanzia, adattamenti, albi 